
\title{
25 Research Square \\ The effect of removable orthodontic appliances on oral microbiota: systematic review
}

\author{
Alessandra Lucchese \\ Universita Vita Salute San Raffaele \\ Marianna Pellegrino ( $\nabla$ mariannapellegrino95@gmail.com ) \\ Eugenio Montini \\ San Raffaele Telethon Institute for Gene Therapy \\ Alessandra Liguori \\ Universita Vita Salute San Raffaele \\ Maurizio Manuelli \\ Universita Vita Salute San Raffaele
}

Universita Vita Salute San Raffaele https://orcid.org/0000-0003-1494-1269

\section{Research article}

Keywords: Oral microbiology, Removable orthodontic appliances, Oral microflora changes, Caries bacteria

Posted Date: December 30th, 2019

DOI: https://doi.org/10.21203/rs.2.19718/v1

License: (c) (1) This work is licensed under a Creative Commons Attribution 4.0 International License.

Read Full License

Version of Record: A version of this preprint was published at Applied Sciences on March 23rd, 2021. See the published version at https://doi.org/10.3390/app11062881. 


\section{Abstract}

Background Removable orthodontic appliances due to plaque accumulation and oral microbe colonization, might be associated with intraoral adverse effects on enamel or periodontal tissues. The present systematic review was carried out to evaluate both qualitatively and quantitatively the microbiological changes occurring during orthodontic therapy with removable orthodontic appliances.

Methods PubMed, Cochrane, EMBASE, Web of Science, Scopus, Ovid Medline, Dentistry \& Oral Sciences Source and Vita-Salute San Raffaele University databases were searched. The research included every article published up to December 2018. The Preferred Reporting Items for Reporting Systematic reviews and Meta Analyses (PRISMA) protocol and the 'Swedish Council on Technology Assessment in Health Care Criteria for Grading Assessed Studies' (SBU) method were adopted to conduct this systematic review.

Results The current study has moderate/high evidence, according with SBU method. It demonstrates that removable appliances do influence the oral microbiota, with significant alterations just 15 days after the beginning of therapy, independently from the type of appliance. Furthermore, the levels of oral pathogens decrease significantly or even returned to pre-treatment levels several months later the therapy end.

Conclusions This review suggests that orthodontic treatment with removable appliances might not induce permanent changes to oral microflora.

Protocol: PROSPERO database registration number CRD42019121762.

\section{Background}

Physiologically the human's oral microflora is composed by a mixture of organisms, which are common also to other anatomical districts. This bacterial charge is extremely complex, being composed by over 700 different species of bacteria [1-5]. Humans are not randomly colonized and the diverse community that makes up the oral microbiome is finely tuned by nature to protect against disease, and it is of great importance to maintain its natural diversity. This particular composition depends on numerous factors, some non-modifiable such as genetics, age, sex, change of dentition [6] and some modifiable, including stress, nutrition, dental treatment and diet of the individual [7-9]. The placement of removable orthodontic appliances creates a favorable environment for the accumulation of microbiota components and food residues, which, in time, may cause caries or exacerbate any pre-existing periodontal disease [10-13]. The appliances, both fixed and removable ones [14,15], may interfere with oral hygiene practice and cover considerable parts of the tooth surfaces, so an increase of the total microbial population as well as an altered microflora have been reported in relation to orthodontic treatment [16].

Once dysbiosis occurs, the goal of treatment should be to restore the lost harmonic balance by maintaining good oral hygiene and modifying lifestyle factors such as diet and smoking. The indiscriminate use of antibiotics for the treatment of oral diseases should be avoided to safeguard the 
beneficial oral microbiota and avoid resistance to antibiotics. For the control of caries, in addition to the use of topical fluoride, measures should be taken to promote the elimination from the acidic environment, through the reduction of the amount and frequency of consumption of sucrose and acidic drinks (even if the latter are sugar-free), together with an integration with agents that can reduce the production of acid and / or promote the generation of alkali in dental plaque. For periodontal diseases, therapeutic strategies should aim to mechanically reduce accumulated biofilm by mechanically removing plaque to levels compatible with oral health. This would reduce the inflammation and flow of GCF and promote a favorable microenvironment to support the formation of a balanced microbiome. The role of the oral microbiome is important not only in the disease, but also in the response of the oral tissues.

Patients need to understand and be aware of the implications for their oral health, when undergoing recommended orthodontic treatment. On the other hand, when the patient accepts to undergo orthodontic treatment, including those using removable orthodontic devices, he should be reminded that it entails a commitment to a higher regimen of attention towards oral hygiene and health in patient's home care $[17,18]$.

The purpose of this review is to investigate the available evidence regarding the association between removable orthodontic appliances and both qualitative and quantitative changes of oral microbiota.

Thus, the clinical research questions proposed are:

- Do removable orthodontic appliances influence the quality and quantity of oral microbiota?

- Which are the effects of removable orthodontic appliances on the different bacterial species in the oral cavity?

\section{Methods}

\section{1 Protocol}

The present study was conducted by the Department of Dentistry at Vita-Salute San Raffaele University of Milan. This systematic review was performed in accordance with the guidelines of the Preferred Reporting Items for Systematic Reviews and Meta-Analyses (PRISMA) statement [19,20]. The analysis' methods and inclusion criteria were specified in advance. No funding was given for the realization of the present review.

\subsection{Search strategy}

The following electronic databases were searched from their respective sources: PubMed, Cochrane Library, Embase, Web of Science, Scopus, Ovid Medline, Dentistry \& Oral Sciences Source and Cochrane Library. In addition, the databases within the library of the Vita-Salute San Raffaele University of Milan were searched. 
To create an appropriate research question and review of the literature the PICOS strategy was used $[21,22]$.

The key words and combinations used in searching the databases were "(Functional appliance OR removable orthodontic appliance OR Frankel appliance OR Bionator OR LM activator OR Twin Block) AND oral microbiology".

Articles published up to 20 December, 2018 were included without language and initial date restriction.

\subsection{Eligibility criteria}

Initially, all articles were selected by title and abstract. Articles present in different databases were considered only once.

In a second moment inclusion and exclusion criteria were applied.

The following inclusion criteria were applied:

1. The microbial analysis had to focus on the quality and quantity of changes in the mouth and not on the appliance and the statistical analysis of the studies had to be adequate [23,24].

2. Only the studies which analyzed functional removable orthodontic appliances were included, in this way space maintainers, aligners or removable retainers were not considered [25-29].

3. Only in vivo, in humans, studies were considered.

For what concern the exclusion criteria, the following were adopted:

1. Patients with systemic diseases or under any condition that could influence oral microbiota or periodontal support tissues;

2. Antibiotic therapy within three months before or during the study;

3. No standardization and training in oral hygiene.

4. Studies that didn't specify the time of collection of samples [30-32].

\subsection{Study selection}

To minimize bias, two review authors, with experience in Oral Microbiology (MM) and Functional Orthodontics (LA), analyzed each selected paper and extracted data independently. Full text was consulted whenever the abstract was missing some information. If data were not clear enough, an attempt was made to contact the author by e-mail. Any disagreement between the two reviewers was resolved by discussion or consultation with a third experienced author (PM).

The Preferred Reporting Items for Reporting Systematic reviews and Meta Analyses (PRISMA) protocol was adopted to report this systematic review $[19,20]$. The article selection process is illustrated in Fig. 1. 


\subsection{Data collection}

The following data was then collected from each included study: author/year of publication, study design, sample size, sample/age/sex, type of appliance, collection time, collection method of analysis, microbial analysis outcome and quality of the study (Table 1).

\subsection{Risk of bias and quality analysis}

The methodological quality is 'the extent to which the design and conduct of a study are likely to have prevented systematic errors (bias)'. Different quality criteria can explain variation in the results of studies included in a systematic review. More rigorously designed (better 'quality') trials are more likely to reach results that are closer to the 'truth' [33]. The 'Swedish Council on Technology Assessment in Health Care Criteria for Grading Assessed Studies'(SBU) method was adopted to report the level of evidence of this systematic review. To minimize the risk of bias during the inclusion of studies in the analysis, the two reviewers (LA and LA) applied independently the SBU criteria. When there was any disagreement concerning the relevance of an article, it was solved by the intervention of a third reviewer (PM). This Protocol organized the articles in three grades according to their methodological quality [34], as Table 2 shows.

Based on the grade of quality, four evidence levels were used, which are explained in the table below (Table 3).

\section{Results}

From the initial 184 articles, 8 were selected [10,35-41].

\subsection{Quality of evidence}

According to the SBU criteria, the evidence of the selected sample is of medium-high level due to the lack of error of measurements analysis for the collection of material from oral sites. Four of the eight articles chosen presented a moderate methodological quality $[10,37,38,40]$ : the major concern regarding these studies is the lack of blinded outcome assessment, diagnostic reliability tests and reproducibility tests. According to the PRISMA method, two articles had a high quality $[35,36]$ and the remaining two were classified as having a low quality $[39,41]$. Due to the absence of homogeneity in the study formulation, a meta-analysis could not be performed and a systematic review was realized instead.

When organizing the data according to pathogens, the following results were obtained:

\subsection{Candida}

All studies detected an increase of Candida spp. concentration during therapy with removable orthodontic therapy [10,36-39]. According to Jabur et al. study, removable orthodontic appliances induced an increase of Candida level up to $13.3 \%$ after an average of 5 weeks and $20 \%$ after a period of 4 months [10]. 
On the contrary the increase in Candida was very low after a period of 3 weeks [36] and 6 months [37].

In Addy's study the Candida prevalence after three weeks from the beginning of treatment resulted to be $46 \%$ in the control group and $52 \%$ of removable appliance wearers [36].

In Arendorf et al. study, noted a prevalence of Candida of $57.6 \%$ for all study subjects, but the $39.4 \%$ of the sample was a prior Candida-carrier, so only $18.2 \%$ became carriers 5 months after starting the therapy. Results of Mc-Nemar test shows highly significant overall increase in Candida prevalence while patients were wearing the appliances $(p<0.001)$, especially in posterior and anterior palatal sites, respectively. However, it was followed by a highly significant fall in number after removal of the appliance $(p<0.001)$, in fact after 5 months form the end of the therapy, only $42.4 \%$ reported Candida colonies. This means that removable orthodontic appliances induced an increase of Candida colonies only of 3\% [38].

An increasing number of microbiological counts of Candida albicans from baseline to one month, three months and six months after the beginning of therapy was observed, with a sudden increase in the number of Candida counts between the baseline and the end of the first month, which was found to be statistically significant $(p<0.001)$ [39].

According with Khanpayeh et al. study, the average number of Candida colonies isolated from saliva after 6 months from the beginning of the therapy with removable appliance $(p=0.001)$ was: Candida albicans $25 \%$, Candida tropicalis 3\%, Candida parapsilosis $2 \%$, Candida krusei $1 \%$ and Candida kefyr $0 \%$. Though, salivary carrier of Candida species decreased with increasing duration of orthodontic treatment [37].

\subsection{Streptococcus mutans}

All three articles $[35,39,41]$ which analysed $S$. mutans colonization of the mouth agreed that removable orthodontic appliances represent a promoting factor for the colonization of the oral cavity by this microorganism.

In Kundu's et al. article a statistically significant increase of $S$. mutans was recorded during orthodontic therapy with removable appliances, from the baseline to six months $(p<0.001)$. Furthermore $S$. mutans bacterial counts were significantly higher than those of Lactobacillus spp. and Candida albicans at alltime intervals (1 - 3 - 6 months) [39].

The study that analyzed different interceptive removable appliances [41], demonstrated a constant increase of Lactobacillus and an increase of $S$. mutans after 15 days, followed by a progressive decrease after 30 and 60 days.

The numbers of $S$. mutans colonies showed a continuous increase during therapy from baseline to one month with statistical significance $(p<0.05)$ [35].

\subsection{Lactobacillus}


All the studies $[10,39,41]$ which quantitatively and qualitatively evaluated the difference of frequency in Lactobacillus spp. demonstrated an increase of the microbiological counts.

Kundu et al. and Topaloglu et al. studies both suggested that the microscopic counts of Lactobacillus $s p p$. increased significantly during orthodontic treatment with removable appliances from the baseline (before appliance placement), to follow-up visits at 1 month, 3 months, and 6 months, these were found to be statistically significant $(p<0.05)[39,41]$.

Jabur et al. noted an increase (6.66\%) in Lactobacillus spp. after 4 months of therapy, too [10].

\subsection{Moraxella catharralis}

According to Jabur et al. study, this pathogen was found in all the included patients, furthermore its oral colonization incredibly increased with removable orthodontic appliances. After a mean of 5 weeks from the appliance use, Moraxella prevalence was of $73.33 \%$ and after 5 months it arrived to $100 \%$ [10].

\subsection{Staphylococcus epidermidis}

S. epidermidis colonization of the mouth also appears to be influenced by the use of removable appliances. As Jabur et al. stated, in patients using these devices the percentage increased up to $40 \%$ after an average of 5 weeks and arrived to $60 \%$ after 4 months [10].

\subsection{Streptococcus viridans}

According to Jabur et al. the frequency of $S$. viridans was of $73.33 \%$ after an average of 5 weeks of therapy with removable orthodontic appliances. The frequency increased up to $80 \%$ after 4 months of treatment [10].

\subsection{Others}

The following results revealed that the changes in oral microbiota during treatment with removable orthodontic devices, involved also other bacterial species.

Petti et al. revealed that in supragingival and in subgingival plaque $G+\operatorname{cocci}$ decreased after 6 - 8 weeks and increased at 6-7 months, with final values higher than baseline values. Gingivitis risk indices (bacterial count and G-rods) significantly increased progressively after 6-8 weeks. Among periodontitis risk indices, only supragingival $m$ rods and subgingival Spirochetes significantly increased at 6-7 months. Aggregatibacter actinomycetemcomitans (Aa) prevalence was near zero [40].

The presence of anaerobic bacteria in the subgingival dental plaque was detected with the same density ( $n=15.75 \%)$ at baseline (T0) and at three months (T1), while it increased at nine months (T2) ( $n=$ $17.85 \%)$, but not significantly ( $p>0.05)$. The most important bacteria that cause periodontal tissue loss Aggregatibacter actinomycetemcomitans (Aa), Porphyromonas gingivalis (Pg), Tannerella forsythia (Tf) and Prevotella nigrescens - were not detected in any patients [40]. 


\section{Discussion}

Any appliance or device placement in the oral cavity increases plaque retention sites and influences the overall microflora. Orthodontic therapy with removable appliances also influences the value of $\mathrm{pH}$ by decreasing significantly during therapy. This value however returns to physiological levels just after 6 months from the end of therapy [38]. Furthermore, the buffering capacity of saliva is statistically significantly reduced during therapy [38]. This means that orthodontic treatment may lead to a modification in the oral microflora, increasing the concentration of cariogenic bacteria in dental plaque and saliva [35]. The microorganisms which increase more, in fact, are Streptococcus mutans, [35, 39, 41] and Lactobacillus spp. [10, 39, 41], but also Staphylococcus epidermidis [10], Moraxella catharralis [10], and subgingival Spirochetes [40] each one with statistical significance. An increase of Candida was also detected $[10,36,38]$ in particular within the first month, with statistical significance $(p<0.001)$ [39]. It was also interesting to notice that the concentration of Candida species in saliva decreased with increasing orthodontic treatment duration [37].

It was also demonstrated that previous orthodontic therapies do not alter the response of the oral microbiota to removable orthodontic treatment. Indeed, patients who have already undergone orthodontic treatment $[28,29]$ have the same alterations in microorganisms of those who never wore orthodontic appliances [24].

These results emphasize that removable orthodontics appliances, when inserted in the oral cavity, begin to accumulate plaque. However, it is not possible to understand whether the accumulation of plaque could be associated with the type of orthodontic appliances material, because most studies in the literature do not specify this. The process that causes an increase in microorganisms load could be strictly related to the appliance surface roughness as well as the time spent in the oral cavity. Generally, removable orthodontics appliances are made of heat-setting plastic or acrylic resin, which are both microporous and rough materials. The introduction of smoother surface removable devices could be less resilient for microorganisms and more biocompatible.

Therefore, it is essential that both patients and healthcare professionals embrace the concept of a balanced oral microbiota and its importance to oral and systemic health. Treatment sessions should include prevention strategies, such as advice on oral hygiene practices, nutrition and smoking, especially during treatment with removable orthodontic devices. As a first approach, active maintenance of oral health, rather than disease management, should be the primary concern for patients. Oral health professionals can achieve this by educating patients on appropriate life choices and the application of effective plaque control techniques, that preserve dental biofilms at levels compatible with oral health. This approach maintains the beneficial properties of resident microbiota and reduces the risk of dental disease due to excessive plaque build-up, qualitative changes of oral microflora and fungal infections.

\section{Conclusions}


According to our systematic review, removable orthodontic appliances influence qualitatively and quantitatively the oral microflora. A significant alteration of microbiota, in fact, is registered just 15 days after the beginning of orthodontic therapy with removable devices, independently from the type of appliance. Nevertheless, the levels of oral pathogens decrease significantly or even returned to pretreatment levels several months later the therapy end (from 3 weeks to 12 months) [35]. Though, this review suggest that orthodontic removable appliances might not induce permanent changes to oral microbiota.

Orthodontic treatment with removable appliances causes an increase of S. mutans, Lactobacillus spp. and Candida spp. in oral mouth, but it has not been possible to understand if there is an association between these alterations of oral microorganisms and the orthodontic materials adopted.

Further studies are required to determine the amount of time required, after the end of therapy, by oral microbiota to return to original values [42] and to investigate if biocompatible materials all have the same effects on it.

\section{List Of Abbreviations}

PICOS:Patient, Intervention, Comparator, Outcome, and Study design; PRISMA:Preferred Reporting Items for Systematic Reviews and Meta-Analyses; SBU:The Swedish Council on Technology Assessment in Health Care; Aa:Aggregatibacter actinomycetemcomitans, Pg:Porphyromonas gingivalis, Tf:Tannerella forsythia.

\section{Declarations}

- Ethics approval and consent to participate: Not applicable.

- Consent for publication: Not applicable.

- Availability of data and materials: all data generated or analysed during this study are included in this published article.

- Competing interests: the authors declare that they have no competing interests.

- Funding: Not applicable.

- Authors' contributions: AL conceived the work. MP and AL collected, analysed and interpreted data regarding microbial oral disease and the removable oral appliances. EM and MM gave a critical revision of the article. MP and AL were the major contributors in writing the manuscript. All authors read and approved the final manuscript.

- Acknowledgements: Not applicable.

\section{References}

1. Bordenstein SR, Theis KR. Host biology in light of the microbiome: Ten principles of holobionts and hologenomes. PLoS Biol. 2015:13:e1002226. 
2. Kilian M, Chapple ILC, Hannig M, Marsh PD, Meuric V, Pedersen AM, et al. The oral microbiome - an update for oral healthcare professionals. Br Dent J. 2016;221:657-66.

3. Sender R, Fuchs S, Milo R. Are We Really Vastly Outnumbered? Revisiting the Ratio of Bacterial to Host Cells in Humans. Cell. 2016;164:337-40.

4. Cho I, Blaser MJ. The human microbiome: At the interface of health and disease. Nat Rev Genet. 2012;13:260-270.

5. Relmand DA. The human microbiome and the future practice of medicine. JAMA. 2015;314:1127-8.

6. Lucchese A, Manuelli M. Prognosis of third molar eruption: a comparison of three predictive methods. Prog Orthod. 2003;4:4-19.

7. Rigo L, Viscioni A, Franco M, Lucchese A, Zollino I, Brunelli G, et al. Overdentures on implants placed in bone augmented with fresh frozen bone. Minerva Stomatol. 2011;60:5-14.

8. Palmieri A, Zollino I., Clauser L, Lucchese A, Girardi A, Farinella F, et al. Biological Effect of Resorbable Plates on Normal Osteoblasts and Osteoblasts Derived From Pfeiffer Syndrome. J Craniofac Surg. 2011;22:860-863.

9. Lucchese A, Manuelli M, Albertini P, Asperio P, Gastaldi G. Treatment of Severe Maxillary Hypoplasia With Combined Orthodontics and Distraction Osteogenesis. J Craniofac Surg. 2018;29:970-972.

10. Jabur SF. Influence of Removable Orthodontic Appliance on Oral Microbiological Status. Fac Med Baghdad. 2008;50:199-202.

11. Zachrisson BU. Oral hygiene for orthodontic patients: Current concepts and practical advice. Am J Orthod. 1974;66:487-497.

12. Gandini P, Schiavi A, Manuelli M, Camassa D. Epidemiological survey of caries occurrence in school age children. Mondo Ortod. 1989;40:63-72.

13. Lucchese A, Porcù F, Dolci F. Effects of Various Stripping Techniques on Surface Enamel. J Clin Orthod. 2001;35:691-5.

14. Lucchese A, Sfondrini MF, Manuelli M, Gangale S. Fixed space maintainer for use with a rapid palatal expander. J Clin Orthod. 2005;39:557-8.

15. Lucchese A, Manuelli M, Bassani L, Albertini P, Matarese G, Perillo L, et al. Fiber reinforced composites orthodontic retainers. Minerva Stomatol. 2015;64:323-33.

16. Lucchese A, Bondemark L, Marcolina M, Manuelli M. Changes in oral microbiota due to orthodontic appliances: a systematic review. J Oral Microbiol.. 2018;10:1476645.

17. Manuelli M. On line is the future. Prog Orthod 2012;13:20.

18. Al Makhmari SA, Kaklamanos EG, Athanasiou AE. Short-term and long-term effectiveness of powered toothbrushes in promoting periodontal health during orthodontic treatment: A systematic review and meta-analysis. Am J Orthod Dentofac Orthop. 2017;152:753-766.

19. Liberati A, Altman DG, Tetzlaff $\mathrm{J}$ et al. The PRISMA statement for reporting systematic reviews and meta-analyses of studies that evaluate health care interventions: explanation and elaboration. $\mathrm{J}$ Clin Epidemiol. 2009;62:e1-34. 
20. Moher D, Liberati A, Tetzlaff J, Altman DG. Preferred reporting items for systematic reviews and meta-analyses: the PRISMA statement. Int J Surg. 2010;8:336-41.

21. Cooke A, Smith D, Booth A. Beyond PICO: the SPIDER tool for qualitative evidence synthesis. Qual Health Res. 2012;22:1435-43.

22. Schardt C, Adams MB, Owens T, Keitz S, Fontelo P. Utilization of the PICO framework to improve searching PubMed for clinical questions. BMC Med Inform Decis Mak. 2007;7:16.

23. Aydinbelge M, Cantekin K, Herdem G, Simsek H, Percin D, Parkan OM. Changes in periodontal and microbial parameters after the space maintainers application. Niger J Clin Pract. 2017;20:1195-1200.

24. D’Ercole S, Martinelli D, Tripodi D. Influence of sport mouthguards on the ecological factors of the children oral cavity. BMC Oral Health. 2014;14:97.

25. Lucchese A, Carinci F, Brunelli G. Skeletal effects induced by twin block in therapy of class II malocclusion. Eur J Inflamm. 2012;10:83-7.

26. Arikan V, Kizilci E, Ozalp N, Ozcelik B. Effects of fixed and removable space maintainers on plaque accumulation, periodontal health, candidal and enterococcus faecalis carriage. Med Princ Pract. 2015;24:311-7.

27. Levrini L, Mangano A, Montanari P, Margherini S, Caprioglio A, Abbate GM. Periodontal health status in patients treated with the Invisalign $(\circledR)$ system and fixed orthodontic appliances: A 3 months clinical and microbiological evaluation. Eur J Dent. 2015;16:296-307.

28. Farhadian N, Usefi Mashoof R, Khanizadeh S, Ghaderi E, Farhadian M, Miresmaeili A. Streptococcus mutans counts in patients wearing removable retainers with silver nanoparticles vs those wearing conventional retainers: A randomized clinical trial. Am J Orthod Dentofac Orthop. 2016;149:155-60.

29. Türköz Ç, Canigür Bavbek N, Kale Varlik S, Akça G. Influence of thermoplastic retainers on Streptococcus mutans and Lactobacillus adhesion. Am J Orthod Dentofac Orthop. 2012;141:598603.

30. Rego RO, Oliveira CA, dos Santos-Pinto A, Jordan SF, Zambon JJ, Cirelli JA, et al. Clinical and microbiological studies of children and adolescents receiving orthodontic treatment. Am J Dent. 2010;23:317-23.

31. Silva CRG, Oliveira LD, Leão MVP, Jorge AOC. Candida spp. adherence to oral epithelial cells and levels of IgA in children with orthodontic appliances. Braz Oral Res. 2014;28:1-5.

32. Kitada K, Toledo A, Oho T. Increase in detectable opportunistic bacteria in the oral cavity of orthodontic patients. Int J Dent Hyg. 2009;7:121-5.

33. Deeks JJ, Glanville J, Sheldon T. Systemic reviews: CRD's guidance for undertaking reviews in health care. York: University of York Press 2009.

34. Facey K. Health technology assessment (HTA) glossary. Sweden: INAHTA Secretariat, c/o SBU Stockholm. 2006.

35. Batoni G, Pardini M, Giannotti A, Ota F, Giuca MR, Gabriele M, et al. Effect of removable orthodontic appliances on oral colonisation by mutans streptococci in children. Eur J Oral Sci. 2001;109:388-92. 
36. Addy M, Shaw WC, Hansford P, Hopkins M. The effect of orthodontic appliances on the distribution of Candida and plaque in adolescents. Br J Orthod. 1982;9:158-63.

37. Khanpayeh E, Jafari AA, Tabatabaei Z. Comparison of salivary Candida profile in patients with fixed and removable orthodontic appliances therapy. Iran J Microbiol. 2014;6:263-268.

38. Arendorf T, Addy M. Candidal carriage and plaque distribution before, during and after removable orthodontic appliance therapy. J Clin Periodontol. 1985;12:360-8.

39. Kundu R, Tripathi A, Jaiswal J, Ghoshal U, Palit M, Khanduja S. Effect of fixed space maintainers and removable appliances on oral microflora in children: An in vivo study. J Indian Soc Pedod Prev Dent. 2016;34:3-9.

40. Petti S, Barbato E, Simonetti, D'Arca A. Effect of orthodontic therapy with fixed and removable appliances on oral microbiota: A six-month longitudinal study. New Microbiol. 1997;20:55-62.

41. Topaloglu-Ak A, Ertugrul F, Eden E, Ates M, Bulut H. Effect of Orthodontic Appliances on Oral Microbiota-6 Month Follow-up. J Clin Pediatr Dent. 2011;35:433-6.

\section{Tables}

[Due to technical limitations, table 1 could not be displayed here. Please see the supplementary files section to access table 1.]

Table 2. SBU criteria for grading assessed studies.

\section{SBU CRITERIA FOR GRADING ASSESSED STUDIES}

\section{Grade A:}

high value of evidence. All criteria should be met: randomized clinical study or a prospective study with a welldefined control group, defined diagnosis and endpoints, diagnostic reliability tests and reproducibility tests described, blinded outcome assessment.

\section{Grade B:}

moderate value of evidence. All criteria should be met: cohort study or retrospective case series with defined control or reference group, defined diagnosis and endpoints, diagnostic reliability tests and reproducibility tests described.

\section{Grade C:}

low value of evidence. One or more of the conditions below: large attrition, unclear diagnosis and endpoints, poorly defined patient material. 
The table reports the Swedish council on technology assessment in health care (SBU) criteria for grading assessed articles.

Table 3. Evidence level definition.

\begin{tabular}{lll} 
LEVEL & EVIDENCE & DEFINITION \\
\hline $\mathbf{1}$ & Strong & At least two studies assessed at level "A". \\
\hline $\mathbf{2}$ & Moderate & One study with level "A" and at least two studies at level "B". \\
\hline $\mathbf{3}$ & Limited & At least two studies at level "B". \\
\hline $\mathbf{4}$ & Inconclusive & Fewer than two studies at level "B". \\
\hline
\end{tabular}

The table shows the criteria used to define the level of evidence of the selected papers.

\section{Figures}




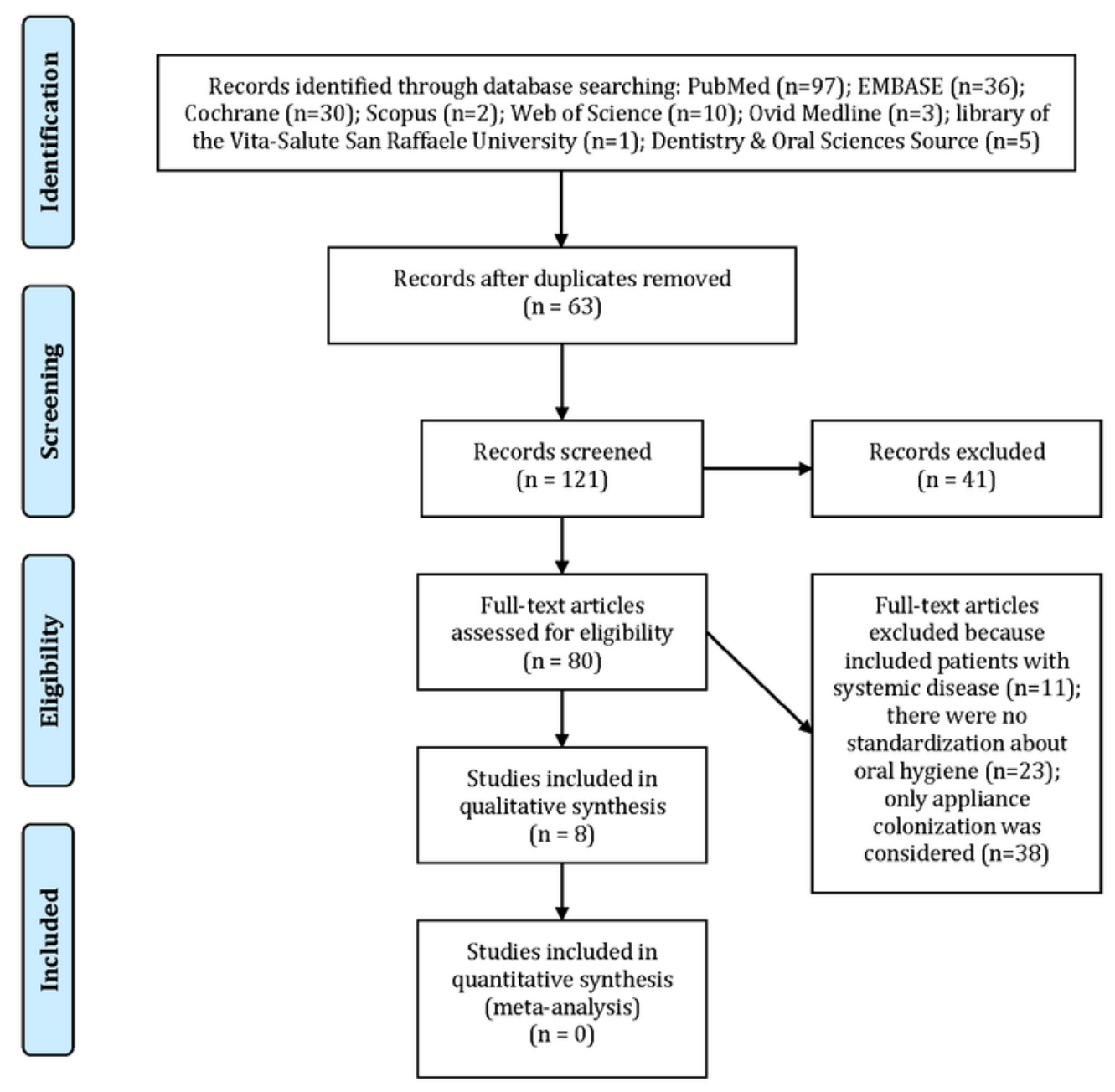

Figure 1

PRISMA 2009: flow diagram of the screening and selection process.

\section{Supplementary Files}

This is a list of supplementary files associated with this preprint. Click to download. 
- Table1.docx

Page 15/15 